$\underline{\text { Research article }}$

\title{
Identification of non-tuberculous mycobacteria isolated from patients at Teaching Hospitals, Kandy and Peradeniya
}

\author{
N P Senanayake ${ }^{1}$, N B Eriyagama ${ }^{1}$, V Thevanesam ${ }^{1}$ \\ Sri Lankan Journal of Infectious Diseases 2016 Vol.6 (1):33-42 \\ DOI: http://dx.doi.org/10.4038/sljid.v6i1.8102
}

\begin{abstract}
Introduction

Non-tuberculous mycobacteria (NTM) are known to cause opportunistic nosocomial infections. The aim of the study was to identify NTM using culture characteristics, biochemical and molecular methods (multiplex PCR) from stored mycobacteria isolated from patients presenting with pulmonary and extrapulmonary tuberculosis at Teaching Hospitals, Kandy and Peradeniya during a 4-year period from 2004 to 2009.
\end{abstract}

\section{Methods}

Forty mycobacterial isolates obtained from 48 samples of sputum, urine, broncho-alveolar lavage and peritoneal fluid were analyzed initially by growth characteristics, followed by biochemical and molecular methods for the presence of NTM. Five main growth characteristics were analyzed, which included rate of growth, growth-temperature relationship, production of pigment, colony morphology and growth on McConkey agar. Species identification of these NTM isolates was by performing biochemical tests. Molecular identification was performed by multiplex-PCR technique on mycobacterial clinical isolates for the rapid identification of NTM.

\section{Results}

Of the 40 mycobacterial isolates, 10 were identified as NTM by using culture characteristics and multiplex PCR. All the NTM isolates were identified to species level by biochemical methods and eight species of NTM were identified. These species, obtained from sterile as well as non-sterile clinical samples were identified as M. scrofulaceum, M. haemophilum, M. chelonae, M. kansasii, M. phlei, M. flavescens, M. gastri and M. vaccae.

\section{Conclusion}

Non-tuberculous mycobacteria were isolated from a wide range of sterile and non-sterile clinical samples and contributed to $25 \%$ of the mycobacterial isolates in this study. The results of culture characteristics were compatible with the molecular identification (multiplex PCR) differentiating Mycobacterium tuberculosis from non-tuberculous mycobacterial strains. M. kansasii and M. gastri were the most common NTM isolated from clinical isolates.

Keywords: non-tuberculous mycobacteria, growth characteristics, biochemical identification, multiplex-PCR 


\section{Introduction}

Non-tuberculous mycobacteria include mycobacterial species other than members of the $M$. tuberculosis complex and M. leprae. NTM (also called environmental, atypical or mycobacteria other than tuberculosis) are a group of human, animal and bird pathogens. ${ }^{1}$ Non-tuberculous mycobacteria are usually found in a variety of environmental reservoirs, which include natural and municipal water, soil, aerosols, protozoans, animals and humans. ${ }^{1}$ There are currently over 125 identified species of NTM. $^{2}$ Non-tuberculous mycobacterial diseases are seen worldwide and the prevalence of NTM diseases are increasing in previously unrecognized populations. ${ }^{1}$ There has been a recent decline in the prevalence of tuberculosis in the developed world and this has been accompanied by an increase in the rate of mycobacterial diseases caused by NTM. ${ }^{3}$

The landscape of diseases caused by non-tuberculous mycobacteria was changed significantly by the emergence of the HIV epidemic in many parts of the world. There has been a significant increase in the incidence of non-tuberculous mycobacterial disease since the first reports of NTM disease in HIV patients in $1982 .{ }^{4}$

Human NTM infections are acquired from environmental exposure. There is no evidence of animal-tohuman or human-to-human transmission of NTM. ${ }^{5}$ Non-tuberculous mycobacteria are known to cause localized or disseminated infections based on local predisposition and /or degree of immunity. ${ }^{6}$ Due to the ubiquitous existence of NTMs in the environment, identifying a causative relationship is dependent on appropriate sampling and strict laboratory practices, as contamination should be excluded. ${ }^{6}$ It is likely that the incidence of NTM infections will increase in the future. ${ }^{1}$

The aim of this study was to determine the proportion and phenotypic/genotypic identification of NTM among mycobacterial clinical isolates obtained from patients presenting with pulmonary and extrapulmonary diseases at Teaching Hospitals, Kandy and Peradeniya.

\section{Methodology}

The study population consisted of stored mycobacterial isolates $(n=40)$ obtained from 48 samples of sputum, urine, broncho-alveolar lavage and peritoneal fluids in patients presenting with pulmonary and extra-pulmonary diseases at Teaching Hospitals, Kandy and Peradeniya during the period January 2004 to January 2009.

Mycobacterial isolates were analyzed initially by growth characteristics followed by biochemical and molecular methods for the presence of NTM. All work with tuberculous material and cultures were conducted inside a class II biological safety cabinet. Initially, microscopic examination of acid-fast smears (by performing Ziehl-Neelson test) was carried out on the 40 isolates to confirm that the growth was mycobacterial and to determine whether contamination with aerobic bacteria was present. ${ }^{7}$ Only uncontaminated isolates were included in the study.

A loopful of growth from each primary culture was placed in a suspension bottle containing $1 \mathrm{ml}$ of distilled water and sterile glass beads. Forty suspension bottles were prepared. Six slopes of Lowenstein-Jensen (LJ) media and two slopes of MacConkey media in universal bottles were inoculated delivering 3 drops of the suspension. ${ }^{7}$ The bottles were incubated under aerobic conditions at $37^{\circ} \mathrm{C} .{ }^{7}$ 


\section{Growth characteristics:}

Five main growth characteristics were analyzed which included, rate of growth, colonial morphologic features, pigment production \& response to light, growth - temperature relationship and growth on MacConkey agar. ${ }^{8}$

\section{Table 1: Colony morphology}

$\begin{array}{ll}\text { Smooth } & \mathrm{S} \\ \text { Rough } & \mathrm{R} \\ \text { Intermediate in roughness } & \mathrm{SR} \\ \text { Thin or transparent } & \mathrm{t} \\ \text { Filamentous extensions } & \mathrm{f}\end{array}$

The culture media were incubated under aerobic conditions at 37 ${ }^{\circ} \mathrm{C}$ and inspected daily for visible colony appearance in order to differentiate between slow (>10days), rapid (<7days) and moderate (7-10days) growers. Five main types of colonial morphologic features were identified as shown in Table 1. Four LJ slopes (two slopes for each test) were incubated at $37{ }^{\circ} \mathrm{C}$ aerobically and inspected daily for six weeks. Two LJ slopes (one covered with black foil paper) were incubated at $37{ }^{\circ} \mathrm{C}$ aerobically in an internally illuminated incubator. These were examined at 3, 7, 14 and 21 days. When the growth was evident on the uncovered LJ slopes, both slopes were examined for pigment production to differentiate between protochromogens, scotochromogens and nonchromogens. Six LJ slopes (two slopes for each temperature range) were incubated at $25^{\circ} \mathrm{C}, 37^{\circ} \mathrm{C}$ and $45^{\circ} \mathrm{C}$ under aerobic conditions and inspected daily for six weeks to assess the growth- temperature relationship.

The M. fortuitum-chelonae complex was differentiated from other species by growth on MacConkey agar without crystal violet as only this NTM complex grows on this medium within five days. A loopful of the broth culture was used to inoculate a plate of MacConkey agar without crystal violet and incubated at $37^{\circ} \mathrm{C}$ aerobically. This was examined after 5 and 11 days for the presence of a visible growth. ${ }^{8}$

\section{Biochemical identification:}

Species identification of NTM was done by performing biochemical tests which included Tween 80 hydrolysis, Sodium chloride tolerance, Tellurite reduction, Iron uptake, Urease test, Catalase test, Nitrate reduction, Pyrazinamidase test and Niacin accumulation. ${ }^{8}$

\section{Molecular identification:}

Multiplex PCR was used for the rapid identification of the genus Mycobacterium, M. tuberculosis complex, M. avium and M.intracellulare. ${ }^{9}$

This method employs a multiplex PCR to identify some common species of mycobacteria from positive cultures and utilizes primers described by Wilton and Cousins (Table 2). ${ }^{9}$ The gene encoding 16S rRNA shows sequence differences that allow for the detection following amplification of a $1030 \mathrm{bp}$ product for Mycobacterium genus, 850 bp product for $M$. intracellulare and 180 bp product for $M$. avium. The gene encoding for the MPB70 protein is utilized to produce a product of $373 \mathrm{bp}$ for the $M$. tuberculosis complex. ${ }^{9}$ The annealing temperature was $60^{\circ} \mathrm{C}$.

Table 2: The sequences of each primer set

\begin{tabular}{|c|c|c|}
\hline & Forward primer & Reverse primer \\
\hline Mycobacterium spp. & AGA GTT TGA TCC TGG CTC AG - 3' & TGC ACA CAG GCC ACA AGG GA - 3’ \\
\hline M. tuberculosis & GAA CAA TCC GGA GTT GAC AA - 3' & AGC ACG CTG TCA ATC ATG TA - 3' \\
\hline M. avium & GAA CAA TCC GGA GTT GAC AA - 3' & ACC AGA AGA CAT GCG TCT TG - 3' \\
\hline M. intracellulare & CCT TTA GGC GCA TGT CTT TA - 3' & ACC AGA AGA CAT GCG TCT TG - 3' \\
\hline
\end{tabular}


DNA extraction: A small amount of growth from the mycobacterial clinical isolates $(<1 \mu L)$ were added to labeled biofuge tubes containing $1 \mathrm{ml}$ of diethylpyrocarbonate- treated distilled water $\left(\mathrm{DepCdH}_{2} \mathrm{O}\right)$ and vortexed. The tubes were centrifuged for 5 minutes at $2500 \mathrm{rpm}$ and the supernatants aspirated completely, taking care not to remove any cell pellet. Two hundred $\mu \mathrm{l}$ of $\operatorname{DepCdH}_{2} \mathrm{O}$ was added to all tubes and vortexed well to re-suspend the pellet and the tubes were sonicated for 15 minutes. The tubes were heated to between $95{ }^{\circ} \mathrm{C}-100{ }^{\circ} \mathrm{C}$ for 15 minutes and the initial preparations diluted to 1:100 with DepC. ${ }^{9}$

Eight $\mu 1$ of the extracted DNA sample was mixed with Qiagen Master mix containing multiple primers (primers for Mycobacterium spp., M. tuberculosis, M. avium and M. intracellulare). ${ }^{9} \mathrm{DEPCdH}_{2} \mathrm{O}$ was used as negative control and the reference strains of each mycobacterial species (M. tuberculosis H37RV, M. avium, M. intracellulare and $M$. kansasii) were used as the positive controls. $^{9}$ Amplification products were analyzed by an Agilent Bio-analyzer 2100 using a disposable chip based gel matrix for amplicon detection and a $2.5 \%$ agarose gel electrophoresis for detection of amplicons. ${ }^{9}$

\section{Results}

Of the 40 mycobacterial isolates tested, 30 (75\%) were slow growers, produced rough colonies, were nonchromogenic and grew only at $37^{\circ} \mathrm{C}$ and were confirmed as M. tuberculosis by PCR. These 30 strains were isolated from sputum samples. The remaining 10 strains $(25 \%)$ were identified as NTM isolates

Eight of the 10 NTM strains were isolated from urine and sputum samples Growth characteristics of NTM isolates are as given in Table 3. . There were rapid and slow growing NTM. Of the NTM isolates six were pigment producers (four scotochromogens and two photochromogens).

Table 3: Growth characteristics of NTM isolates

\begin{tabular}{cccccc}
\hline $\begin{array}{c}\text { Sample } \\
\text { source }\end{array}$ & Rate of growth Pigmentation & $\begin{array}{c}\text { Growth temp. } \\
\text { relationship } \\
\left(25^{\circ} \mathrm{C}, 37^{\circ} \mathrm{C}, 45^{\circ} \mathrm{C}\right)\end{array}$ & $\begin{array}{c}\text { Colony } \\
\text { morphology }\end{array}$ & $\begin{array}{c}\text { Growth on } \\
\text { McConkey agar }\end{array}$ \\
\hline Urine & $\mathrm{Sl}$ (21 days) & $\mathrm{S}$ & 25,37 & $\mathrm{~S}$ & Negative \\
Urine & $\mathrm{Sl}$ (12 days) & $\mathrm{P}$ & 25,37 & $\mathrm{SR}$ & Negative \\
Sputum & $\mathrm{S} 1$ (12 days) & $\mathrm{P}$ & 25,37 & $\mathrm{SR}$ & Negative \\
Sputum & $\mathrm{Sl}$ (9 days) & $\mathrm{N}$ & 25,37 & $\mathrm{~S}$ & Negative \\
BAL & $\mathrm{Sl}$ (9 days) & $\mathrm{N}$ & 25,37 & $\mathrm{R}$ & Negative \\
Peri. fluid & $\mathrm{Ra}$ (5 days) & $\mathrm{N}$ & 25,37 & $\mathrm{~S}$ & Positive \\
Urine & $\mathrm{Ra}$ (4 days) & $\mathrm{S}$ & 25,37 & $\mathrm{R}$ & Negative \\
Sputum & $\mathrm{Ra}$ (5 days) & $\mathrm{S}$ & 25,37 & $\mathrm{~S}$ & Negative \\
Urine & $\mathrm{Sl}$ (14 days) & $\mathrm{N}$ & 25,37 & $\mathrm{R}$ & Negative \\
Sputum & $\mathrm{Sl}$ (10 day & $\mathrm{S}$ & 25,37 & $\mathrm{~S}$ & Negative \\
\hline
\end{tabular}

Peri. fluid Peritoneal fluid; Ra Rapid grower; S1 Slow grower; N Non chromogens; P Photochromogens; S

Scotochromogens; R Rough; Sm Smooth; SR Smooth to rough;

All the NTM isolates grew at $25^{\circ} \mathrm{C}$ and $37^{\circ} \mathrm{C}$. Colony morphology varied from smooth to rough. Only one NTM isolate grew on McConkey agar. 
Growth characteristics of NTM isolates are shown in Table 3 and biochemical and species identification in Table 4. The commonest NTM isolates were M. kansasii and M. gastri.

Table 4: Biochemical and the species identification of NTM clinical isolates:

\begin{tabular}{|c|c|c|c|c|c|c|c|c|c|c|}
\hline 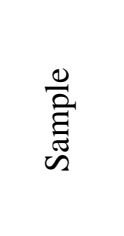 & 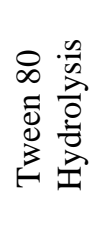 & 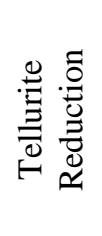 & 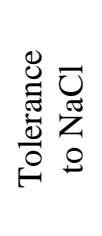 & 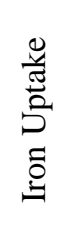 & 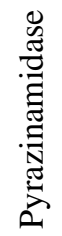 & 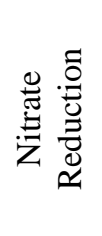 & 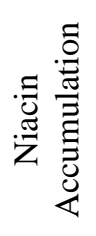 & 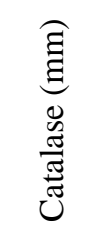 & 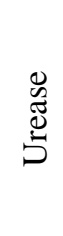 & 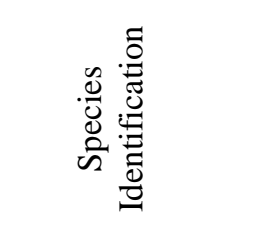 \\
\hline Urine & - & - & - & - & + & - & - & $>45$ & + & M. scrofulaceum \\
\hline Urine & + & + & - & - & - & + & - & $>45$ & + & M. kansasii \\
\hline Sputum & + & + & - & - & - & + & - & $>45$ & + & M. kansasii \\
\hline Sputum & + & + & - & - & - & - & - & $<45$ & + & M. gastri \\
\hline BAL & + & - & - & - & - & - & - & $<45$ & + & M. gastri \\
\hline $\begin{array}{l}\text { Peri. } \\
\text { fluid }\end{array}$ & + & + & - & - & + & - & - & $>45$ & + & $\begin{array}{l}\text { M. chelonae/M } \\
\text { abscessus }\end{array}$ \\
\hline Urine & + & + & + & + & - & + & - & $>45$ & + & M. phlei \\
\hline $\begin{array}{l}\text { Tween } \\
\text { Sputum }\end{array}$ & + & + & - & + & - & + & - & $>45$ & - & M. vaccae \\
\hline Urine & - & - & - & - & + & - & - & $<45$ & - & M. haemophilum \\
\hline Sputum & + & + & + & - & + & + & - & $>45$ & + & M. flavescens \\
\hline
\end{tabular}

All 40 isolates were positive for genus mycobacteria of which $30(75 \%)$ isolates were positive for MTB and $10(25 \%)$ isolates were positive for NTM by multiplex PCR (Table 5).

Table 5: Multiplex PCR of Mycobacterial isolates

\begin{tabular}{cccccc}
\hline $\begin{array}{c}\text { Number of } \\
\text { isolates }\end{array}$ & $\begin{array}{c}\text { Mycobacterium } \\
\text { genus }(1030 \mathrm{bp})\end{array}$ & $\begin{array}{c}\text { M. tuberculosis } \\
(373 \mathrm{bp})\end{array}$ & $\begin{array}{c}\text { M. avium } \\
(180 \mathrm{bp})\end{array}$ & $\begin{array}{c}\text { M. intracellulare } \\
(850 \mathrm{bp})\end{array}$ & $\begin{array}{c}\text { Results of } \\
\text { Multiplex } \\
\text { PCR }\end{array}$ \\
\hline 30 & + & + & - & - & MTB \\
10 & + & - & - & - & NTM \\
\hline
\end{tabular}

\section{Discussion}

Non-tuberculous mycobacteria are saprophytic organisms which are found in soil, water and dust. These organisms are however known to cause a variety of infections, usually in immunocompromised individuals. ${ }^{10}$ Although the incidence of infections caused by NTM is increasing throughout the world, the isolation rate of NTM appears to vary considerably in different parts of the world. ${ }^{11}$ A contributory factor for these differences may be due to differences in transport of specimens and techniques of isolation, in addition to variations in the prevalence of NTM. ${ }^{11}$ 
Although the incidence of tuberculosis has decreased in developed countries, infections due to NTM have shown to be on the rise. However, in some developing countries like India where tuberculosis remains a major health problem, NTM infections are also commonly reported. ${ }^{10}$

The aim of this study was to determine the proportion and identification of NTM in patients presenting with pulmonary and extra-pulmonary tuberculosis at Teaching Hospitals, Kandy and Peradeniya. A previous large scale study from South India on the isolation of NTM from clinical specimens in patients with suspected pulmonary and extra pulmonary tuberculosis, showed that during a study period of six years, $13 \%$ of the specimens grew acid-fast bacilli (AFB). Of these, $96.1 \%$ were M. tuberculosis while $3.9 \%$ were NTM. The disease burden of NTM infections in India is not known. ${ }^{10}$ A study from Zambia enrolled 180 patients and 385 controls. Of the patients, 33\% had only M. tuberculosis isolates in their sputum samples, $7 \%$ had $M$. tuberculosis and NTM, and 11\% had only NTM. In another pilot study from Zambia, high rates of NTM culture-positive sputum samples were obtained in 3 hospitals in 2001. ${ }^{12}$

In our study, 40 mycobacterial isolates were cultured from 48 samples during a 4 year period between January 2004 and January 2009, of which 10 (25\%) were identified as NTM isolates. It is difficult to compare our data on NTM diseases with published data from other countries due to variation in the study periods, selection criteria and the populations studied. In the present study we were able to isolate eight species of NTM in 10 of the 40 isolates from both sterile and non-sterile clinical samples. They include M. scrofulaceum, M. haemophilum, M. chelonae, M. kansasii (two isolates), M. phlei, M. flavescens, M. gastri (two isolates) and M. vaccae.

As NTM organisms are ubiquitous and a possible laboratory contaminant, the isolation of these organisms from specimens should meet objective criteria to confirm their significance. These include, repeated isolation of the same organism from a patient, associated positive clinical and radiological evidence, histopathological confirmation, collection of appropriate specimens directly from the lesions, and isolation from sterile body fluids. The presence of predisposing factors and the immune status of the patient should also be taken into consideration when reporting NTM from clinical specimens. ${ }^{10}$ The diagnosis of NTM disease is obvious when isolation of the organism is from a sterile site such as blood, bone marrow, lymph nodes or synovial fluid. ${ }^{12}$ However, it is difficult a make a firm diagnosis of NTM infections when NTMs are isolated from non-sterile sources such as sputum or bronchoalveolar lavage samples, especially with low colony numbers and in situations where NTM are isolated from only one cultured specimen. In these patients it is challenging to differentiate NTM infections from contamination and colonization. ${ }^{12}$

\section{The clinical significance of the NTM isolates:}

In the current study, M. scrofulaceum, M. haemophilum, M. kansasii and M. phlei were isolated from samples of urine. Although disseminated $M$. scrofulaceum infection is rare even in immunocompromised patients, it has previously been reported in the urine of a healthy man. ${ }^{13}$ There are several reported cases of $M$. haemophilum causing an array of infections in immunocompromised patients in a Cancer Institute in New York. ${ }^{14}$ The clinical syndromes on presentation included skin lesions, arthritis, osteomyelitis and lung diseases. ${ }^{14}$ There have been no previous case reports of isolation of this organism from urine in the published literature. It is possible that the urinary isolate in the current study is due to environmental contamination.

The second most common opportunistic non-tuberculous mycobacterial infection associated with AIDS is M. kansasii. M. kansasii is not usually isolated from environmental sources unlike other 
NTM. M. kansasii was isolated from a sample of urine in the current study. Disseminated M. kansasii infection has been previously reported in a 40 years old male patient associated with pancytopenia with granulomata in the kidney and liver, where the organism was isolated from urine and tissue biopsies. ${ }^{15}$

M. phlei has been isolated very rarely in human infections. It was isolated from the peritoneal fluid of a patient who presented with recurrent episodes of chronic ambulatory peritoneal dialysis-associated peritonitis. ${ }^{16}$ As there are no reported cases of urine infections caused by $M$. phlei, the presence of this organism in urine is most likely to be a result of contamination.

Most NTM peritonitis cases are reported in patients undergoing peritoneal dialysis. There are reported cases of $M$. chelonae peritonitis associated with continuous ambulatory peritoneal dialysis (CAPD). ${ }^{17}$ The isolation of $M$. chelonae in a sample of peritoneal fluid in the current study is probably of clinical significance.

M. kansasii, M. flavescens, $M$. gastri, and M. vaccae were isolated from samples of sputum and BAL in the current study, although the most commonly reported pathogen, M. avium from these sites was not isolated in this study. The commonest presentation of $M$. kansasii infection is a chronic pulmonary infection that mimics pulmonary tuberculosis. There are many reported cases worldwide of pulmonary infections where $M$. kansasii has been repeatedly isolated from sputum samples. There are 40 to 70 cases per year in Great Britain and in the Los Angeles area (USA), the incidence was 2.4 cases/100,000 inhabitants, including HIV positive patients. ${ }^{18}$ As $M$. kansasii is an unlikely environmental contaminant, the isolation of this organism from sputum is likely to be of clinical importance.

M. flavescens is usually described as nonpathogenic in humans but occasionally reported as the cause of serious infectious complications. Clinical information available was inadequate to decide whether the presence of this organism in sputum was due to environmental contamination or infection.

Mycobacterium gastri is infrequently implicated as a cause of infection in clinical practice. Few reports have been published about infections caused by this organism, which included respiratory infections. ${ }^{19}$ Isolation of this organism from sputum in the current study may be of clinical significance as it has been isolated from sputum in cases of pulmonary infection.

There are reported cases of $M$. vaccae being associated with pulmonary infections and soft-tissue infections in four patients in the southern United States. ${ }^{20}$ The isolation of this organism from a sample of sputum could be of clinical significance.

Laboratory services are very important in the diagnosis, management and epidemiological investigation of TB and other mycobacterial diseases. ${ }^{21}$ The presumptive differentiation between MTB and NTM can be made by growth characteristics and by microscopic observation of cording formation on ZiehlNeelson (ZN) stain. $^{21}$ Although identification and differentiation by microscopic and culture characteristics alone is not definitive and also time consuming ${ }^{22}$, clinical and radiological diagnostic methods, along with direct microscopy and culture is the mainstay of investigation of suspected mycobacterial infections in resource limited-settings. ${ }^{22}$

Limitations of using morphology, growth characteristics and biochemical profile for identification of mycobacteria are well known. The slow growth rate of members of this genus results in serious delays in reporting, which has clinical and public health consequences. Low specificity resulting in misidentification and identification of biochemically unreactive or new species remains a challenge. ${ }^{23,24}$ 
The gold standard for the identification of mycobacteria is the DNA sequence analysis of the $16 \mathrm{~S}$ rRNA gene region, which however requires expensive equipment, reagents and trained personnel, all of which increases the cost of the test. ${ }^{25}$

Correct identification of mycobacterial species is essential for accurate diagnosis of NTM infections and to prevent incorrect and often unnecessary treatment with anti-tuberculous chemotherapy. Identification by culture characteristics is time consuming. Therefore, rapid and definitive identification of the species in the sample is of prime importance for appropriate antibiotic therapy and public health measures. ${ }^{22,26}$ However, resource deficient countries often overlook infections owing to NTM because of the unavailability of required resources. Although efforts to improve the diagnosis of these infections are needed in such situations, assessment of the optimal use of currently available diagnostic tools remains important.

Three modalities of identification, namely, culture, bio-chemical and molecular methods were used in the current study to assess both the frequency of isolation of NTM as well as the accuracy of identification of clinical mycobacterial isolates. Culture characteristics and biochemical identification using a limited number of tests were able to separate $M$. tuberculosis from NTM accurately in the small sample studied. Species identification was more challenging and time consuming and needs to be studied further as early identification of diseases caused by mycobacteria is needed for patient management, both to decide on optimal therapy and also to reduce implementation of unnecessary infection control measures. ${ }^{27}$

\section{Conclusion}

The capacity to identify mycobacteria up to species level using biochemical and molecular methods is required as one fourth of isolates were NTM, necessitating different therapeutic as well as prevention strategies. Mycobacterial laboratory services need to be developed so that regular documentation and reporting of NTM with sensitivity profiles are available to clinicians managing such patients.

\section{Limitations of the study}

As the study was done on stored isolates, clinical data was not available for consideration of significance of the isolates.

\section{Conflicts of Interest}

The authors have no conflicts of interest.

\section{References}

1. Todd P., Christie A., Joseph O. Health impacts of environmental mycobacteria. Clinical Microbiology Reviews. 2004; 17(1):98-106. http://dx.doi.org/10.1128/CMR.17.1.98-106.2004

2. Falkinham J., Norton C. Factors influencing numbers of $M$. avium, M. intracellulare and other mycobacteria in drinking water distributing systems. Applied and Environmental Microbiology. 2001; 67(3):1225-1231. http://dx.doi.org/10.1128/AEM.67.3.1225-1231.2001

3. Piersimoni C., Scarparo C. Pulmonary infection associated with nontuberculous mycobacteria in immunocompetent patients. Lancet Infectious Diseases. 2008; 8(5):323-324. http://dx.doi.org/10.1016/S1473-3099(08)70100-2

4. Falkinham JO. Epidemiology of infection by nontuberculous mycobacteria. Clinical Microbiology Reviews.1996; 9(2):177-215. http://dx.doi.org/10.1164/ajrccm/136.2.344

5. Griffith DE., Aksamit T. Diagnosis, treatment and prevention of nontuberculous mycobacterial 
diseases. American Journal of Respiratory Critical Care Medicine. 2007; 175(7):367-416. http://dx.doi.org/10.1164/rccm.200604-571ST

6. Katoch VM. Infections due to nontuberculous mycobacteria. Indian Journal of Medical Sciences. 2004; 120(4):290-304. http://dx.doi.org/10.1186/s40409-015-0009-8

7. Barrow GI and Faltham RKA (eds). Cowan and Steel's Manual for the Identification of Medical Bacteria ( $3^{\text {rd }}$ ed). Cambridge: Cambridge University Press., 1999.

8. Roberts GD., Koneman EW and Kim YK (eds). Manual of Clinical Microbiology (5 ${ }^{\text {th }}$ ed). Washington D.C: American Society for Microbiology., 1991.

9. Wilton $\mathrm{S}$ and Cousins D. Detection and identification of multiple mycobacterial pathogens by DNA amplification in a single tube PCR: Methods and Application 1992: pp 269-273.

10. Jesudasan MV., Gladstone P. Non-tuberculous mycobacteria isolated from clinical specimens at a tertiary care hospital in South India. Indian Journal of Medical Microbiology. 2005; 23(3):172175. http://dx.doi.org/10.4103/0255-0857.16589

11. Chauhani MM. Non-tuberculous mycobacteria isolated from an epidemiological survey in rural population of Bangalore district. Indian Journal of Tuberculosis. 1993; 40(4):195-197. http://dx.doi.org/10.4103/0974-777X.77305

12. Buijtels PC., Vander Sande MAB., Graaff CS. Nontuberculous mycobacteria, Zambia. Emerging Infectious Diseases Journal. 2009; 15(2):242-249. http://dx.doi.org/10.3201/eid1502.08006

13. Hsueh PR., Hsiue TR., Jarn JJ. Disseminated infection due to Mycobacterium scrofulaceum infection in an immunocompetent host. Clinical Infectious Diseases. 1996; 22:159-161. http://dx.doi.org/10.1093/clinids/22.1.159

14. Shah MK., Sebti A., Kiehn TF., Sepkowitz KA. Mycobacteruim haemophilum in immunocompromised patients. Clinical Infectious Diseases. 2001; 33(3):330-337. http://dx.doi.org/10.1086/321894

15. William J., Listwan MD., Donald D., Roth MD. Disseminated M. kansasii infection with pancytopenia and interstitial nephritis. Annals of Internal Medicine. 1975; 83(1):70-73 http://dx.doi.org/10.7326/0003-4819-83-1-70

16. Paul E., Devarajan P. Mycobacterium phelei peritonitis: a rare complication of chronic peritoneal dialysis. Pediatric Nephrology. 1997; 12(1):67-68. http://dx.doi.org/10.1007/s004670050407

17. Lee KF., Chen HH., Wu CJ. M. chelonei peritonitis in a patient on Peritoneal Dialysis. Renal Failure. 2008; 30(3):335-338. http://dx.doi.org/10.1080/08860220701861185

18. Morrone N., Cruvinel MC., Oliveira LML., Goncalves C. Lung disease caused by Mycobacterium kansasii. Journal de Pneumologia. 2003; 29(6):341-346. . http://dx.doi.org/10.1590/S0102-35862003000600005

19. Velayati AA., Mohammad RB., Parissa F. Mycobacterium gastri causing disseminated infection in children of same family. Paediatric Pulmonology. 2005; 39(30):284-287.

http://dx.doi.org/10.1002/ppul.20186

20. Hachem R., Kenneth IR., Rolston VI. Cutaneous and pulmonary infections caused by Mycobacterium vaccae. Clinical Infectious Diseases. 1996; 23(1):173-175.

http://dx.doi.org/10.1016/j.vaccine.2014.03.047

21. Giampaglia CNS., Martins NC., Chimara C. Differentiation of Mycobacterium tuberculosis from other Mycobacteria with Nitrobenzoic acid using MGIT960. International Journal of Tuberculosis Lung Diseases. 2007; 11(7):803-807. http://dx.doi.org/10.4103-0857.96697

22. Richardson ET., Samson D., Banaei N. Rapid identification of Mycobacterium tuberculosis and Non-tuberculous mycobacteria by multiplex, real-time PCR. Journal of Clinical Microbiology. 2009; 47(5):1497-1502. http://dx.doi.org/10.1128/JCM.01868-08

23. Cook VJ., Christine Y., Wolfe J., Pauls R., Kabani A. Conventional methods versus 16S ribosomal DNA sequencing for identification of nontuberculous mycobacteria: Cost analysis. Journal of Clinical Microbiology. 2003; 41(3):1010-1015. 
http://dx.doi.org/10.1128/JCM.41.3.1010-1015.2003

24. Mondragon-Barreto M., Vasquez-Chacon CA., Barren-Rivero C., et al. Comparison among three methods for mycobacteria identification. Salaud Publica de Mexico. 2000; 42(6):484-489. http://dx.doi.org/10.1590/S0036-36342000000600003

25. Wang H., Yue J., Han M., Yang J.,Zhao Y. Rapid method for identification of six common species of mycobacteria based on multiplex SNP analysis. Journal of Clinical Microbiology. 2010; 48(1):247-250. http://dx.doi.org/10.1128/JCM.01084-09

26. Garima K., Varma-Basil M., Pathak R.,et al. Are we looking infections owing to non-tuberculous mycobacteria during routine conventional laboratory investigations? International Journal of Mycobacteriology. 2012; 1(4):207-211. http://dx.doi.org/10.1016/j.ijmyco.2012.10.005

27. Kox LFF., Jensen HM., Kuijper S., Kolk AHJ. Multiplex PCR Assay for immediate identification of the infecting species in patients with mycobacterial diseases. Journal of Clinical Microbiology. 1997; 35(6):1492-1495. http://dx.doi.org/10.1016/j.femsec.2004.09.015 